\title{
DEPRESSÃO EM MULHERES IDOSAS: UMA REVISÃO BIBLIOGRÁFICA
}

\author{
Manoela Vieira de Souza ${ }^{1}$, Chennyfer Dobbins Abi Rached ${ }^{2}$ \\ 1 Graduanda de Enfermagem. Universidade Nove de Julho - UNINOVE. São Paulo - Brasil - Email: \\ São Paulo - Brasil \\ 2 Doutora em Saúde Coletiva; Mestre em Economia da Saúde pela Universidade Federal de São \\ Paulo (UNIFESP); Docente do Programa de Mestrado Profissional em Gestão em Sistemas de Saúde - \\ Universidade Nove de Julho - UNINOVE. São Paulo - Brasil email: chennyferr@yahoo.com.br ORCID: \\ https://orcid.org/0000-0002-4499-3716
}

\section{RESUMO}

A depressão, considerada o "mal do século", é uma doença que afeta o humor, gerando um sentimento de tristeza profunda onde não há um motivo justificável para ocorrer. Este trabalho teve como objetivo "elencar cuidados e intervenções da assistência de enfermagem à paciente idosa com depressão". A metodologia utilizada foi a revisão de literatura do tipo narrativa, realizada na base de dados: Biblioteca Virtual em Saúde (BVS), Scientific Eletronic Library Online (SCIELO) e Google Acadêmico, utilizando os seguintes descritores: Depressão, Idosas, Assistência de enfermagem na depressão, Saúde do Idoso, Diagnósticos de Enfermagem. Uma das ações de enfermagem que é importante realizar no idoso com depressão são as escalas: Mini Exame do Estado Mental (MEEM ou mini-mental), Escala de Depressão de Geriátrica (GDS) de Yesavage e Escala de Atividades Básicas de Vida Diária (Katz), para identificar o estado da saúde mental, o grau de complexidade em que o paciente se encontra e se o mesmo tem suspeita de depressão. Conclui-se que os cuidados e intervenções de enfermagem são importantes fatores de ajuda para melhorar a qualidade de vida dos idosos, contribuindo assim para aumentar a expectativa de vida dos mesmos.

Palavras-chave: Depressão, Idosas, Assistência de enfermagem na depressão, Saúde do Idoso, Diagnósticos de Enfermagem.

\section{ABSTRACT}

Depression, considered the "evil of the century", is a disease that affects mood, generating a deep sense of sadness where there is no justifiable reason to occur. The objective of this study was to "list care and interventions of nursing care for the elderly patient with depression". The methodology used was the literature review of the narrative type, carried out in the database: Virtual Health Library (VHL), Scientific Eletronic Library Online (SCIELO) and Google Scholar, using the following descriptors: Depression, Elderly, Nursing care in Depression, Elderly Health, Nursing Diagnoses. One of the nursing actions that is important to perform in the elderly with depression are the scales: Mini Mental State Examination (MEEM or minimental), Yesavage Geriatric Depression Scale (GDS) and Basic Daily Life Scale (Katz ), to identify the state of mental health, the degree of complexity in which the patient is and if the patient suspects depression. It is concluded that nursing care and interventions are important help factors to improve the quality of life of the elderly, contributing to increase their life expectancy.

Keywords: Depression, Elderly, Nursing care in depression, Elderly Health, Nursing Diagnoses. 


\section{INTRODUÇÃO}

A depressão, considerada o "mal do século", é uma doença que afeta o humor, gerando um sentimento de tristeza profunda onde não há um motivo justificável para ocorrer. Além de que pode ser uma doença sintomática ou assintomática. Pode afetar pessoa de qualquer classe social, assim como se relaciona com a qualidade de vida ${ }^{1}$.

A qualidade de vida quando contemplada por aspectos físicos, emocionais, sociais e capacidade funcional, pode inibir a depressão ${ }^{1}$.

De acordo com as Pirâmides Etárias realizadas pelo IBGE (Instituto Brasileiro de Geografia e Estatística) no Brasil a cada 10 anos, a população deixou de ser jovem e se tornou adulta, mais especificamente, houve um crescimento considerável da população idosa ${ }^{3}$.

Essa doença está associada à morbilidade e mortalidade não só com o que condiz ao suicídio, mas também por aumentar o prognóstico das doenças do tipo crônicas chamadas de “orgânicas”, principalmente doenças coronárias e DM tipo 2 (Diabetes mellitus) ${ }^{8}$.

A depressão tem afetado consideravelmente mulheres da terceira idade. Um exemplo a ser notado é que, mulheres que praticam exercícios físicos, possuem uma qualidade de vida melhor do que as sedentárias, o que por si só já é um fator para não vedar a depressão².

"Mal do século", expressão utilizada durante o romantismo movimento caracterizado por ser uma reação do neoclassicismo. De acordo com a definição encontrada no livro Dicionário de Termos Literários, de Moisés Massaud, o Mal do Século é definido como "Pessimismo extremo, em face do passado e do futuro, sensação de perda de suporte, apatia moral, melancolia difusa, tristeza, culto do mistério, do sonho, da inquietude mórbida, tédio irremissível, sem causa, sofrimento cósmico, ausência da alegria de viver, fantasia desmesurada, atração pelo infinito, "vago das paixões", desencanto em face do cotidiano, desilusão amorosa, nostalgia, falta de sentimento vital, depressão profunda, abulia, resultando em males físicos, mentais ou imaginários que levam à morte precoce ou ao suicídio".

\section{OBJETIVO}

Elencar cuidados e intervenções da assistência de enfermagem à paciente idosa com depressão. 


\section{METODOLOGIA}

O presente estudo trata-se de uma pesquisa de revisão de literatura do tipo narrativa. A seleção dos artigos será realizada no período de fevereiro a dezembro de 2018, como base de pesquisa, a Biblioteca Virtual em Saúde (BVS), por meio do site: http://regional.bvsalud.org/php/index.php; Google Acadêmico, por meio do site: https://scholar.google.com.br/ e Scientific Eletronic Library Online (SCIELO), por meio do site: http://www.scielo.org/php/index.php. Utilizando como assuntos principais: Depressão, Idosas, Assistência de enfermagem na depressão, Saúde do Idoso, Diagnósticos de Enfermagem. Os critérios de inclusão dos artigos definidos, inicialmente, para a presente revisão serão: artigos em língua portuguesa, com textos completos disponíveis nas bases de dados citadas acima, no período compreendido entre 2014 a 2018; artigos publicados cuja metodologia adotada permita obter evidências sobre os temas Depressão, Idosas, Assistência de enfermagem na depressão. A seguir, os dados serão transcritos e armazenados no Microsoft Office Word. A apresentação dos resultados será realizada em forma de texto.

\section{DESENVOLVIMENTO}

O aumento da população idosa nos últimos tempos tem motivado cada vez mais a surgirem pesquisas para melhor atender esse grupo social, esses estudos prezam a qualidade de vida considerando o fator idade/envelhecimento, onde é levado em conta a vulnerabilidade desse grupo em todos os aspectos susceptíveis (socioeconômico, espiritual, hospitalização por motivos de doenças/quedas) $)^{6}$.

De acordo com a literatura, a assistência ao idoso deve ser preferencialmente em casa, se ele for hospitalizado, deve ser preparado para que saia o mais breve possível. Sendo que os cuidados realizados pela equipe de saúde sempre serão voltados para manter qualidade de vida, fazendo assim a manutenção da vida, para que o paciente possa se possível, passar a realizar suas tarefas do dia a dia de forma a não necessitar de auxílio?

A depressão é a doença mental que atinge a terceira idade com mais frequência. Essa enfermidade afeta a qualidade de vida e é considerada um importante fator de risco para demências ${ }^{4}$.

Os transtornos mentais, incluindo a depressão, são doenças que afetam a população desde muito antigamente, os primeiros registros são datados por volta de 2600 a.C. De acordo com a história, a partir de estudos de Hipócrates (460-370 a.C.) e seus pupilos é que a tristeza persistente foi então chamada de melancolia. Mas só no século XIX é que começaram a surgir estudos mais profundos sobre o tema, e têm surgido cada vez mais diagnósticos/tratamento relacionados à depressão, só então passou a se chamar depressão ${ }^{6}$. 
De acordo com a literatura, durante a vida, a depressão afeta de 10 a $25 \%$ das mulheres e de 5 a $12 \%$ dos homens, sendo que não tem relação com a classe social, educação, etnias, estado civil, ou seja, pode afetar qualquer pessoa sem um motivo justificável para acontecer ${ }^{6}$.

$\mathrm{O}$ tratamento da depressão nas mulheres idosas tem por finalidade assegurar qualidade de vida, diminuir o risco de suicídio, o sofrimento psíquico e melhorar o bem-estar geral ${ }^{4}$.

É importante investigar aspectos psicológicos e psicossociais com bastante ênfase, a fim de observar se existe a possibilidade de alguma outra doença e/ou o uso de fármacos (remédios para insônia, antiinflamatórios, anti-hipertensivos, entre outros) estarem relacionados aos sintomas da depressão, levando ao surgimento da mesma ${ }^{4}$.

De acordo com a literatura, a depressão tem seus sintomas divididos em quatro tipos, sendo eles: estado de humor, neurovegetativos, cognitivos e psicóticos, como podemos ver na tabela a seguir 4 :

\begin{tabular}{|c|c|c|c|}
\hline \multicolumn{4}{|c|}{ SINTOMAS DA DEPRESSÃO NO IDOSO } \\
\hline $\begin{array}{c}\text { Sintomas do estado de } \\
\text { humor }\end{array}$ & $\begin{array}{c}\text { Sintomas } \\
\text { Neurovegetativos }\end{array}$ & Sintomas cognitivos & $\begin{array}{l}\text { Sintomas } \\
\text { psicóticos }\end{array}$ \\
\hline $\begin{array}{l}\text { - Deprimido/disfórico } \\
\text { - Irritabilidade } \\
\text { - Tristeza } \\
\text { - Desânimo } \\
\text { - Sentimento de } \\
\text { abandono } \\
\text { - Sentimento de } \\
\text { inutilidade } \\
\text { - Diminuição da auto- } \\
\text { estima } \\
\text { - Retraimento } \\
\text { social/solidão } \\
\text { - Anedonia e } \\
\text { desinteresse } \\
\text { - Ideias } \\
\text { autodepreciativas } \\
\text { - Ideias de morte } \\
\text { - Tentativas de suicídio }\end{array}$ & $\begin{array}{l}\text { - Inapetência } \\
\text { - Emagrecimento } \\
\text { - Distúrbio do sono } \\
\text { - Perda da energia } \\
\text { - Lentificação } \\
\text { psicomotora } \\
\text { - Inquietação } \\
\text { psicomotora } \\
\text { - Hipocondria } \\
\text { - Dores inespecíficas }\end{array}$ & $\begin{array}{l}\text { - Dificuldade de } \\
\text { concentração e } \\
\text { memória } \\
\text { - Lentificação do } \\
\text { raciocínio }\end{array}$ & $\begin{array}{l}\text { - Ideias } \\
\text { paranoides } \\
\text { - Delírios de ruína } \\
\text { - Delírios de } \\
\text { morte } \\
\text { - Alucinações } \\
\text { mandativas de } \\
\text { suicídio }\end{array}$ \\
\hline
\end{tabular}


Outro sintoma frequente em pessoas com depressão é a procrastinação. O simples ato de deixar para outra hora algo que se pode realizar naquele momento e depois sentir a vergonha, a culpa, o estresse, a sensação de perda de produtividade pode desencadear a depressão em si.

Em se tratando da fisiopatologia são encontradas algumas alterações em diversas regiões encefálicas: há um aumento de volume e atividade do córtex pré-frontal, amígdalas, córtex anterior do cíngulo e hipocampo. Há um hiperativismo do eixo hipotálamo-hipófisesuprarrenal associado ao receptor glicorticoide. $\mathrm{O}$ foco da investigação são as pós-sinapses desencadeadas pelo nível de monoaminas. O efeito dos antidepressivos pode ser explicado pelo tempo que a neurogênese (formação de novos neurônios) demora a ocorrer ${ }^{8}$.

\section{AÇÕES DE ENFERMAGEM}

Uma das ações de enfermagem que é importante realizar no idoso com depressão são as escalas: Mini Exame do Estado Mental (MEEM ou mini-mental), Escala de Depressão de Geriátrica (GDS) de Yesavage e Escala de Atividades Básicas de Vida Diária (Katz), mostradas nas tabelas a seguir ${ }^{10}$ :

\begin{tabular}{|l|l|}
\hline \multicolumn{2}{|c|}{ MINI EXAME DO ESTADO MENTAL } \\
\hline $\begin{array}{l}\text { Orientação: 10 pontos } \\
\text { Identificar: }\end{array}$ & $\begin{array}{l}\text { - Ano/estação do ano/dia da semana mês/dia do mês/ } \\
\text { local/andar/bairro/cidade/estado }\end{array}$ \\
\hline Registro: 3 pontos & - Memorizar: "copo, mala, carro" \\
\hline Atenção e cálculo: 5 pontos & $\begin{array}{l}\text { - Subtrair 7 de 100, e sucessivamente por 5 vezes } \\
\text { - Soletrar "mundo" de trás para frente }\end{array}$ \\
\hline Memória de evocação: 3 pontos & - Repetir: "copo, mala, carro" \\
\hline Linguagem & $\begin{array}{l}\text { - Nomear "lápis e relógio" - 2 pontos } \\
\text { - Repetir: "nem aqui, nem ali, nem lá" - 1 ponto } \\
\text { - Seguir comando em 3 etapas: "pegue este papel com a } \\
\text { mão direita, dobre ao meio e ponha no chão" - 3 pontos } \\
\text { - Seguir comando escrito: "feche os olhos" - 1 ponto } \\
\text { - Escrever sentença com sujeito, verbo e predicado- } 1 \\
\text { ponto } \\
\text { - Copiar o desenho- 1 ponto }\end{array}$ \\
\hline
\end{tabular}




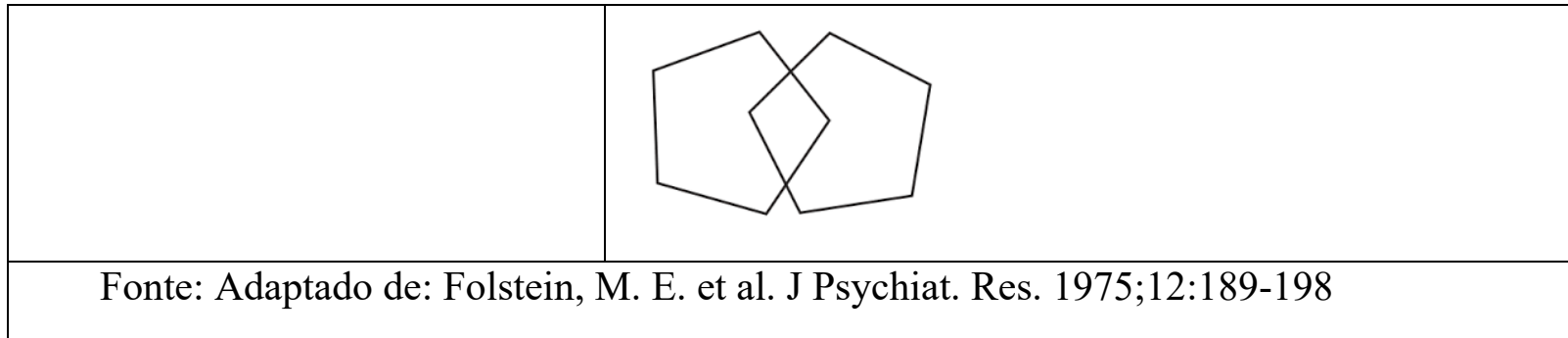

O MEEM (Mini Exame do Estado Mental) serve para identificar o estado da saúde mental do paciente como orientação em tempo, espaço e pessoa, registrar/memorizar, ter atenção, conseguir nomear objetos, repetir frases e seguir comandos ${ }^{10}$.

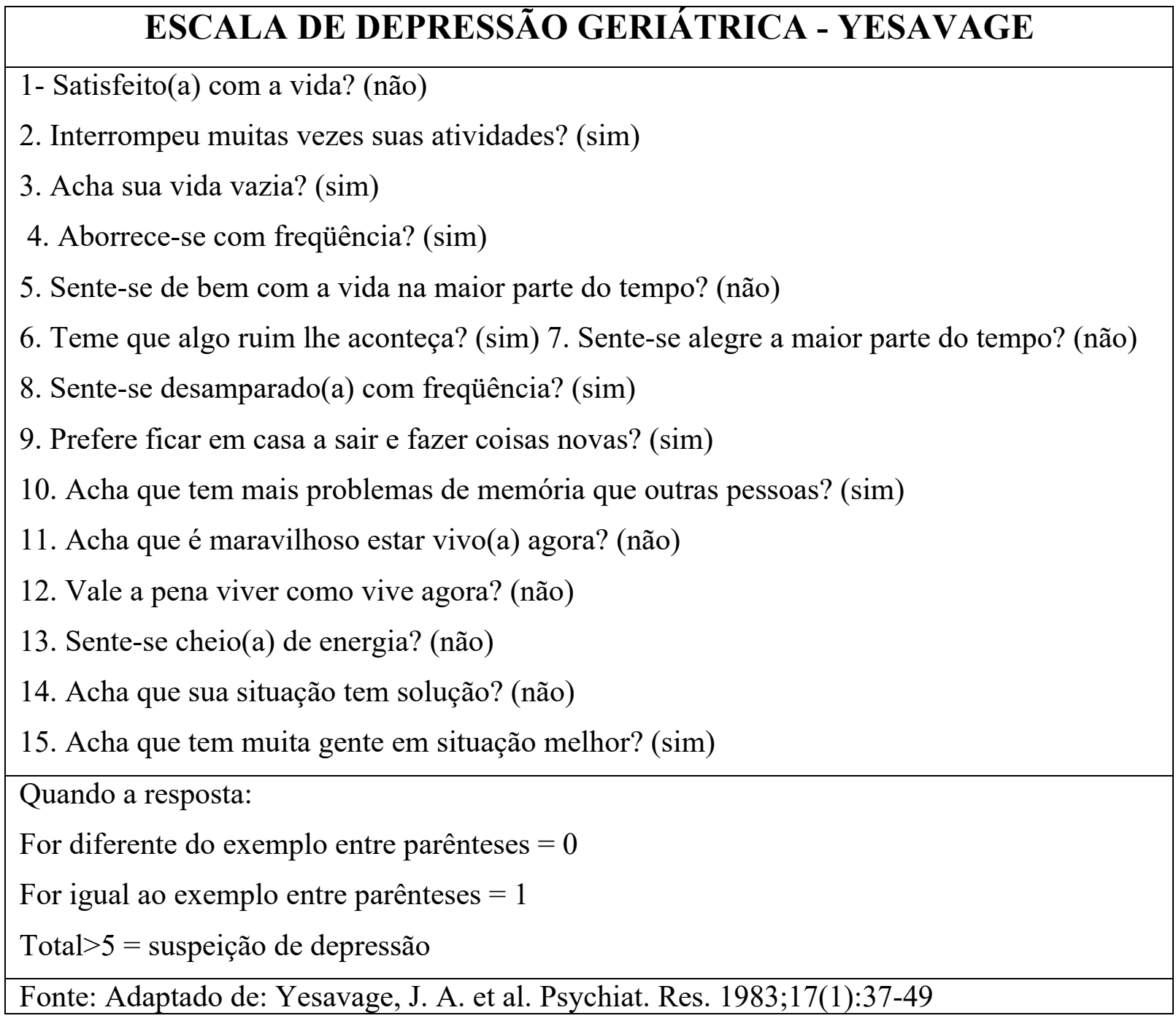

A Escala de Depressão Geriátrica (GDS) de Yesavage é aplicada nos pacientes com 60 anos ou mais e serve para identificar se o idoso tem suspeição de depressão ${ }^{10}$. 


\begin{tabular}{|l|}
\hline \multicolumn{1}{|c|}{ ESCALA DE ATIVIDADES BÁSICAS DE VIDA DIÁRIA (Katz) } \\
\hline 1. Banho \\
I. não recebe assistência \\
A. assistência para uma parte do corpo \\
D. não toma banho sozinho \\
2. Vestuário \\
I. veste-se sem assistência \\
A. assistência para amarrar sapatos \\
D. assistência para vestir-se \\
3. Higiene pessoal \\
I. vai ao banheiro sem assistência \\
A. recebe assistência para ir ao banheiro \\
D. não vai ao banheiro para eliminações fisiológicas \\
4. Transferência \\
I. deita, levanta e senta sem assistência \\
A. deita, levanta e senta com assistência \\
D. não levanta da cama \\
5. Continência \\
I. controle esfincteriano completo \\
A. acidentes ocasionais \\
D. supervisão, uso de cateter ou incontinente \\
6. Alimentação \\
I. sem assistência \\
A. assistência para cortar carne/manteiga no pão \\
D. com assistência, ou sondas, ou fluidos IV \\
Resultado: \\
I. Independência \\
A. Dependência parcial \\
D. Dependência total \\
\hline Fonte: Adaptado de: Katz, S. et al.. JAMA 1963;185(12):914-916. \\
\hline
\end{tabular}

A escala de Atividades Básicas de Vida Diária (Katz) é aplicada para saber em qual grau de complexidade de cuidados o paciente se encontra. Se no teste as respostas resultarem mais na letra I do que nas outras, o paciente é independente, ou seja, não necessita de auxílio para realizar suas Atividades de Vida Diárias (AVDs). Se a Resposta se concentrar na letra A, ele 
possui dependência parcial, como por exemplo, assistência para amarrar os sapatos, passar manteiga, cortar carne, auxílio para ir ao banheiro. E, se o resultado estiver mais concentrado na letra $\mathrm{D}$, esse paciente é considerado Dependente total, necessitando de auxílio para realizar todas as suas AVDs, como escovar os dentes, pentear o cabelo, tomar banho, vestir-se, comer, neste caso, o paciente não levanta da cama ${ }^{10}$.

A depressão na terceira idade é tratável, porém o diagnóstico de depressão pode ser um desafio e acabar sendo confundido com alguma demência e vice-versa pelo fato de apresentarem sintomas iguais e/ou parecidos - déficit de concentração, apatia, autonegligência, déficit no autocuidado, lentificação psíquica e na memória e mudanças no comportamento e personalidade -, além de que a depressão pode ser um dos sintomas de demência. Por isso é importante saber distinguir, para isso, existem algumas características que as diferenciam, como mostrados na tabela a seguir?:

\section{Características clínicas que contribuem para o diagnóstico diferencial entre depressão com déficits cognitivos e demência}

\begin{tabular}{|l|l|}
\hline Demência com sintomas depressivos & Depressão com déficits cognitivos \\
\hline Início insidioso & Início agudo \\
Sintomas geralmente de longa duração & Sintomas geralmente de curta duração \\
Flutuações do humor e do comportamento & Humor persistentemente deprimido \\
Justificativas dos erros cometidos & Respostas do tipo “não sei” \\
Minimização dos déficits pelo paciente & (Hiper) valorização dos déficits \\
Déficits cognitivos relativamente estáveis & Flutuação dos déficits cognitivos \\
\hline $\begin{array}{l}\text { Fonte Neto JG, Tamelini MG, Forlenza OV. Diagnóstico diferencial das demências. Rev. } \\
\text { Psiq. Clín. 2005. 32 (3); 119-130. }\end{array}$ \\
\hline
\end{tabular}

Para pacientes com depressão podem-se citar alguns Diagnósticos de Enfermagem de acordo com o livro NANDA. Sendo eles':

- Autonegligência relacionada a transtorno psiquitátrico, pois a paciente poderá apresentar dificuldades relacionadas às AVDs (Atividades de Vida Diárias) como, por exemplo, autocuidado prejudicado, onde algumas intervenções sugeridas sãos: assistência no autocuidado (auxílio no banho e vestir-se/arrumar-se, auxílio na higiene oral estimular deambulação) $)^{5}$.

- Controle Emocional Instável relacionado a transtorno psiquiátrico, alteração na autoestima, transtorno do humor e perturbação emocional, intervenções sugeridas: escutar ativamente, encaminhar para o profissional de saúde mental (psicólogo, terapeuta) ${ }^{5}$. 
- Isolamento Social relacionado à alteração no estado mental, intervenções sugeridas: terapia de grupo, terapia recreacional, apoio familiar e ao cuidador ${ }^{5}$.

- Risco de Síndrome do Idoso Frágil, cujos fatores de risco são: depressão, isolamento social, tristeza, redução da energia, apoio social insuficiente, morar sozinho, estilo de vida sedentário, atividade física diária inferior a recomendada para o gênero e a idade. Intervenções sugeridas: Aconselhamento, escutar ativamente, orientar sobre os benefícios de não morar sozinho, orientar a família quanto ao apoio e à moradia ${ }^{5}$.

Podem-se citar também alguns outros Diagnósticos de Enfermagem sendo eles: Insônia, Atividade de Recreação Deficiente relacionada, Estilo de Vida Sedentário, Falta de Adesão, Desesperança, Risco ou Baixa Autoestima Crônica ou Situacional, Processos Familiares Disfuncionais, Desempenho de Papel Ineficaz, interação Social Prejudicada, Risco ou Relacionamento Ineficaz, Risco ou Síndrome do Estresse por Mudança, Ansiedade, Enfrentamento Ineficaz, Negação Ineficaz, Risco de Planejamento de Atividade Ineficaz, Regulação do Humor Prejudicada, Risco de Resiliência Comprometida, Tristeza Crônica, etc $^{5}$.

\section{CONSIDERAÇÕES FINAIS}

Nas últimas décadas foi observado o crescente número da população idosa, pois esse grupo tem buscado cada vez mais se conscientizar quantos aos cuidados com a saúde para melhorar a qualidade de vida, o que tem contribuído significativamente para diminuir a mortalidade nessa faixa etária gerando assim maior expectativa de vida. No entanto, são as mulheres que mais buscam ajuda profissional para cuidar da saúde. No presente estudo, notase que uma das doenças que tem acometido bastante a terceira idade é a depressão. A enfermagem desempenha um importante papel no cuidado de pessoas idosas com depressão, como por exemplo, auxiliando o paciente no autocuidado (pentear o cabelo, auxílio no banho e alimentação, entre outros) até que ele seja capaz de realizar suas tarefas sem ajuda. Outra ação de enfermagem é utilizar escalas para identificar o estado da saúde mental, o grau de complexidade em que o paciente se encontra e se o mesmo tem suspeita de depressão. Conclui-se que os cuidados e intervenções de enfermagem são importantes fatores de ajuda para melhorar a qualidade de vida dos idosos, contribuindo assim para aumentar a expectativa de vida dos mesmos. 


\section{REFERÊNCIAS}

1. Rufine MC, Silva AA, Formiga NS, Melo GF. Perfil psicológico de gênero, qualidade de vida e depressão: proposta de um modelo causal em mulheres idosas. Psicol. Argum. 2014. 32(79), Supl. 1, 31-41.

2. Gonçalves FB, Araújo APS, Júnior JRAN, Oliveira DV. Qualidade de vida e indicativos de depressão em idosas praticantes de exercícios físicos em academias da terceira idade na cidade de Maringá (PR). Revista Saúde e Pesquisa. 2015. Set-dez; 8(3), 557-567.

3. 6. Projeções e estimativas da população do Brasil e das Unidades da Federação. Instituto Brasileiro de Geografia e Estatística. Disponível em:

https://www.ibge.gov.br/apps/populacao/projecao/

4. F. Stella, S. Gobbi, Danilla I. Corazza \& J. L. R. Costa. Depressão do Idoso e Atividade Física. Motriz, Rio Claro, Ago/Dez 2002. 8(3), 91-98.

5. Diagnósticos de Enfermagem da NANDA. Artmed. 2015-2017.

6. Garcia A, et al. A depressão e o processo de envelhecimento. Ciências e cognição. 2006. $7(1), 111-121$.

7. Marin MJS, Barbosa PMK, Takitanel MT. Diagnósticos de Enfermagem mais frequentes em idosas hospitalizadas em unidade de clínica médica e cirúrgica. Revista Brasileira de Enfermagem. Brasília. Out/dez 2000. 53(4), 513-523.

8. Lage JT. Neurobiologia da Depressão. Revista: Acta Médica Portuguesa Abril, 2010. 1-24.

9. Neto JG, Tamelini MG, Forlenza OV. Diagnóstico diferencial das demências. Rev. Psiq. Clín. 2005. 32(3), 119-130.

10. Ferrari JF, Dalacorte RR. Uso da Escala de Depressão Geriátrica de Yesavage. Scientia Medica, Porto Alegre. Jan/mar 2007. 17(1), 3-8. 